\author{
(๑ А.Ю. Бабенко ${ }^{*}$, А.А. Мосикян', И.Е. Макаренко², В.В. Леушева², Е.В. Шляхто'
}

${ }^{1}$ Национальный медицинский исследовательский центр им. В.А. Алмазова, Санкт-Петербург ${ }^{2} \mathrm{OOO} \mathrm{ГЕРОФАРМ,} \mathrm{Санкт-Петербург}$

ОБОСНОВАНИЕ. Ингибиторы дипептидилпептидазы 4 типа (иДПП-4), обладая инкретиновой активностью, воздействуют на один из основных патогенетических механизмов сахарного диабета 2 типа (СД2). Эвоглиптин является новым препаратом класса иДПП-4 с уникальными для класса характеристиками фармакокинетики, эффективность и безопасность которого в монотерапии была изучена ранее в плацебо-контролируемых испытаниях.

ЦЕЛЬ. Оценить эффективность и безопасность эвоглиптина в сравнении с ситаглиптином в международном двойном слепом рандомизированном контролируемом испытании у больных СД2 с неудовлетворительным контролем гликемии на монотерапии метформином. Сравнить полученные результаты в российской и корейской популяциях.

МЕТОДЫ. Нами была использована база данных исследования ЭВО-КОМБИ, в которой содержались данные российских и корейских участников исследования (в соотношении 1:4). Всего в исследование 1:1 были рандомизированы пациенты $(\mathrm{n}=281)$, получавшие монотерапию метформином в дозе не менее 1000 мг/сут (142 - в группу эвоглиптина 5 мг, 139 - в группу ситаглиптина, 100 мг). Исследование имело параллельный дизайн, продолжительность терапии составила 24 нед. Первичной конечной точкой было абсолютное изменение уровня гликированного гемоглобина $\left(\mathrm{HbA}_{1 \mathrm{c}}\right)$ через 24 нед по сравнению с исходным значением. Для доказательства не меньшей эффективности эвоглиптина по сравнению с ситаглиптином было необходимо, чтобы верхняя граница 95\% двустороннего доверительного интервала (ДИ) для средней разницы между группами изменения уровня $\mathrm{HbA}_{1 c}$ на 24-й неделе по сравнению с исходными значениями не превышала 0,35\%. Дополнительно проводился анализ подгрупп.

РЕзУЛЬтАТЫ. В группе пациентов, принимавших эвоглиптин, изменение уровня НbА

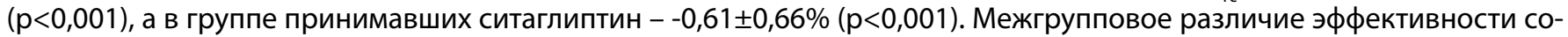
ставило 0,03\% [95\% ДИ:-0,14;0,19\%], что ниже установленной границы 0,35\% и доказывает не меньшую эффективность эвоглиптина по сравнению с ситаглиптином. Наблюдалась тенденция к большей эффективности обоих препаратов в южно-корейской субпопуляции $(p=0,030)$, однако снижение $\mathrm{HbA}_{1 с}$ было сопоставимым ( $\left.p=0,657\right)$. Оба препарата хорошо переносились. Нежелательные явления при приеме обоих препаратов наблюдались преимущественно со стороны желудочно-кишечного тракта (ЖКТ), при этом количество нежелательных явлений (НЯ) было сопоставимо ( $>0,05)$ между препаратами, а НЯ со стороны ЖКТ регистрировались чаще у пациентов из Южной Кореи ( Тяжелых гипогликемических эпизодов зарегистрировано не было. Частота развития легких гипогликемических эпизодов, зарегистрированных в течение 24 нед, была сопоставима между группами ( $p=0,365)$ и составила 0,7\% в группе эвоглиптина и 5,2\% в группе ситаглиптина.

ЗАКЛЮЧЕНИЕ. В настоящем исследовании продемонстрированы не меньшая эффективность и сопоставимая безопасность препарата эвоглиптин в дозе 5 мг один раз в день по сравнению с ситаглиптином 100 мг один раз в день. Профиль эффективности и безопасности эвоглиптина был сопоставим в российской и корейской популяциях.

КЛЮЧЕВЫЕ СЛОВА: иДПП-4; эвоглиптин; ситаглиптин; $\mathrm{HbA}_{1 с}$

\title{
EFFICACY AND SAFETY OF EVOGLIPTIN VERSUS SITAGLIPTIN AS ADD ON TO METFORMIN ALONE IN A COMBINED RUSSIAN-KOREAN POPULATION. EVO-COMBI TRIAL
}

\author{
Alina Y. Babenko ${ }^{1 *}$, Anna A. Mosikian¹, Igor E. Makarenko², Victoriya V. Leusheva², Evgeny V. Shlyakhto ${ }^{1}$
}

${ }^{1}$ National Almazov North-West Medical Research Centre, Saint-Petersburg, Russia

${ }^{2}$ Geropharm, Saint-Petersburg, Russia

BACKGROUND: Dipeptidyl-peptidase-4 inhibitors (iDPP-4) are pathogenically targeted drugs for diabetes mellitus type 2 (T2DM). Evogliptin is a new member of iDPP-4 class. The drug has the longest half-elimination period among the class, and its efficacy and safety as monotherapy have been already studied in placebo-controlled randomized clinical trials.

AIMS: To study efficacy and safety of evogliptin as compared to sitagliptin in T2DM patients with unsatisfying glycemic control with metformin monotherapy via a multinational double blind randomized controlled trial. To compare the study results in Russian and Korean subpopulations. 
MATERIALS AND METHODS: We used a combined Russian-Korean database (1:4) of EVO-COMBI trial. 281 adult T2DM patients administered metformin alone (at least $1000 \mathrm{mg} /$ day) were randomized 1:1 to add on evogliptin (142 patients) or sitagliptin (139 patients) for 24 weeks once daily. The primary endpoint was change in glycated hemoglobin (HbA1c) level at Week 24 as compared to baseline. Non-inferiority was concluded if the upper limit of the 2-sided 95\% confidence interval for the HbA1c difference between treatments was $<0.35 \%$. Subgroup analysis for between-subpopulation difference in treatment effect was also conducted.

RESULTS: The mean between-group difference was $0.03 \%$ [95 \% Cl: $-0.14 ; 0.19 \%$, that confirms non-inferiority of evogliptin (mean $\mathrm{HbA} 1 \mathrm{c}$ decrease $-0.58 \pm 0.70 \%, \mathrm{p}<0.001$ ) to sitagliptin (mean HbA1c decrease $-0.61 \pm 0.66 \%, p<0.001$ ). Evogliptin and sitagliptin both tend to be more effective in South Korean subpopulation in terms of fasting plasma glucose lowering $(p=0.030)$, however $\mathrm{HbA1c}$ decrease in subpopulations was comparable $(p=0.657)$. Both drugs were well tolerated in both subpopulations. Adverse effects were associated mostly with gastrointestinal disorders, and the frequency was comparable between treatment groups ( $>0.05$ ). Gastrointestinal adverse effects were registered more often in Korean patients $(p=0.014)$. There were no severe hypoglycemia. Frequency of mild hypoglycemia was comparable between evogliptin and sitagliptin ( $0.7 \%$ and $5.2 \%$, respectively, $\mathrm{p}=0.365)$.

CONCLUSIONS: Evogliptin $5 \mathrm{mg} /$ day is non-inferior to sitagliptin $100 \mathrm{mg} /$ day in T2DM patients with unsatisfying glycemic control with metformin monotherapy. Safety profile is also comparable. Efficacy-safety profile of evogliptin is comparable in Russian and South Korean subpopulations.

KEYWORDS: iDPP-4; evogliptin; sitagliptin; $\mathrm{HbA}_{1 \mathrm{c}}$

В современных алгоритмах лечения сахарного диабета 2 типа (СД2) ранняя интенсификация с добавлением второго препарата при недостаточной эффективности метформина и ранняя комбинированная терапия для пациентов с $\mathrm{HbA}_{1 c}<7,5 \%$ стали важными опциями достижения и сохранения гликемического контроля. Оптимальными признаны те комбинации, которые воздействуют на основные патогенетические механизмы СД2: инсулинорезистентность (метформин) [1] и нарушение секреции инсулина и глюкагона, на которую оптимальное воздействие оказывают препараты с инкретиновой активностью, в частности ингибиторы дипептидилпептидазы 4 типа (иДПП-4) [2].

В настоящее время В РФ зарегистрирован целый ряд лекарственных препаратов из группы иДПП-4: ситаглиптин, саксаглиптин, вилдаглиптин, линаглиптин и алоглиптин. Для оптимизации выбора препаратов в условиях наличия нескольких представителей класса важно иметь информацию по сравнению эффективности и безопасности отдельных препаратов класса между собой. В то время как для всех перечисленных препаратов были неоднократно представлены результаты клинических исследований, оценивавших их эффективность как в монотерапии [3-7], так и в комбинации с другими препаратами [8-12], прямые сравнительные исследования различных глиптинов носят единичный характер. Сравнение препаратов в основном представлено в мета-анализах. В ряде мета-анализов отмечена несколько большая эффективность ситаглиптина и вилдаглиптина по сравнению с другими препаратами этой группы как в монотерапии [13], так и в комбинации с метформином [14].

В мета-анализе Craddy и соавт., сравнившем эффективность всех выше упомянутых иДПП-4 (алоглиптин, линаглиптин, саксаглиптин, ситаглиптин и вилдаглиптин) (табл. 1) [15], было продемонстрировано сопоставимое абсолютное снижение уровня $\mathrm{HbA}_{1 с}$ в пределах 0,45-0,59\% при сопоставимом риске развития гипогликемического эпизода. Эффективность сочетания различных иДПП-4 и метформина также была сопоставима (см. табл. 1). При этом, согласно данным мета-анализа, прием иДПП-4 в качестве терапии второй линии на фоне приема метформина по сравнению с монотерапией метформином позволял достичь дополнительного снижения $\mathrm{HbA}_{1 c}$ в среднем на 0,48-0,70\%. По результатам настоящего мета-анализа были также получены данные о нейтральности иДПП-4 в отношении массы тела как в монотерапии, так и в сочетании с метформином (см. табл. 1) [15]. В то же время следует иметь в виду, что валидность результатов мета-анализов эффективности иДПП-4 ограничена тем, что выраженность гипогликемического эффекта иДПП-4 прямо коррелирует с исходным уровнем $\mathrm{HbA}_{1 с}$ в связи с глюкозозависимым влиянием препаратов на секрецию инсулина, т.е. абсолютное снижение $\mathrm{HbA}_{1 с}$ зависит от его исходного уровня и индекса массы тела (ИМТ) [16]. В вышеупомянутом мета-анализе в исследования также включались пациенты с разной медианой массы тела, что могло повлиять на результаты (см. табл. 1).

Таким образом, сложность непрямых сравнений эффективности отдельных препаратов обусловлена и влиянием других факторов, детерминирующих эффективность данных препаратов - этническая принадлежность [17], возраст пациента [18, 19], длительность течения диабета [20], выраженность инсулинорезистентности [21] и другие.

Все это свидетельствует в пользу необходимости проведения прямых сравнительных исследований, которые в настоящее время носят единичный характер (ситаглиптин и саксаглиптин [22], ситаглиптин и вилдаглиптин [23]). Эти прямые сравнения продемонстрировали либо сопоставимую [23]), либо лучшую [22] эффективность ситаглиптина и вилдаглиптина. Это позволяет рассматривать их в качестве эталонных препаратов, в основном ситаглиптин, зарегистрированный в большинстве стран мира, в прямых сравнительных исследованиях по оценке эффективности вновь регистрируемых представителей класса иДПП-4.

Эвоглиптин - новый селективный ингибитор ДПП-4, обеспечивающий сопоставимое с ситаглиптином (>80\%) ингибирование фермента в течение 24 ч [24]. Пиковая концентрация в крови достигается через 5 ч после 
Таблица 1. Сравнительный анализ эффективности отдельных ингибиторов дипептидил пептидазы 4 в монотерапии и комбинации с метформином, все значения - среднее взвешенное (95\% доверительный интервал). Адаптировано из Craddy и соавт. [15]

\begin{tabular}{|c|c|c|c|c|c|c|}
\hline Препарат & $\begin{array}{l}\text { Исходный } \\
\text { HbA }_{1 c^{\prime}} \% *\end{array}$ & $\begin{array}{c}\text { Абсолютное } \\
\text { снижение } \\
\text { HbA }_{1 c^{\prime}} \% \\
\end{array}$ & $\begin{array}{c}\text { Вероятность } \\
\text { достижения } \\
\text { HbA }_{1 c}<7 \%\end{array}$ & $\begin{array}{c}\text { Абсолютный } \\
\text { риск развития } \\
\text { гипогликемии }\end{array}$ & $\begin{array}{l}\text { Исход- } \\
\text { ный } \\
\text { вес, кг* }\end{array}$ & $\begin{array}{c}\text { Абсолютное } \\
\text { снижение мас- } \\
\text { сы тела, кг }\end{array}$ \\
\hline \multicolumn{7}{|c|}{ В монотерапии } \\
\hline Алоглиптин 25 мг & 8,00 & $\begin{array}{c}-0,58 \\
(-0,83 ;-0,33)\end{array}$ & $\begin{array}{c}40 \% \\
\text { (34-59\%) }\end{array}$ & $\begin{array}{c}0,13 \% \\
(0,003-0,7 \%)\end{array}$ & 67,7 & $\begin{array}{c}-0,17 \\
(-0,60 ; 0,23)\end{array}$ \\
\hline Линаглиптин 5 мг & 8,13 & $\begin{array}{c}-0,58 \\
(-0,81 ;-0,35)\end{array}$ & $\begin{array}{c}34 \% \\
(19-53 \%)\end{array}$ & $\begin{array}{c}0,8 \% \\
(0,003-4,2 \%)\end{array}$ & 79,1 & $\begin{array}{c}-0,12 \\
(-0,62 ; 0,38)\end{array}$ \\
\hline Саксаглиптин 5 мг & 8,59 & $\begin{array}{c}-0,45 \\
(-0,75 ;-0,15)\end{array}$ & $\begin{array}{c}25 \% \\
(11-44 \%)\end{array}$ & $\begin{array}{c}0,88 \% \\
(0,062-3,8 \%)\end{array}$ & 86,6 & - \\
\hline Ситаглиптин 100 мг & 7,96 & $\begin{array}{c}-0,59 \\
(-0,75 ;-0,43)\end{array}$ & $\begin{array}{c}37 \% \\
(24-51 \%)\end{array}$ & $\begin{array}{c}0,29 \% \\
(0,046-0,97 \%)\end{array}$ & 85,1 & $\begin{array}{c}0,20 \\
(-0,18 ; 0,60)\end{array}$ \\
\hline Вилдаглиптин 50 мг × 2 раза & 8,49 & $\begin{array}{c}-0,52 \\
(-0,71 ;-0,31)\end{array}$ & $\begin{array}{c}39 \% \\
(24-55 \%)\end{array}$ & $\begin{array}{c}0,37 \% \\
(0,043-1,4 \%)\end{array}$ & 89,5 & $\begin{array}{c}0,33 \\
(-0,12 ; 0,80)\end{array}$ \\
\hline \multicolumn{7}{|c|}{ В комбинации с метформином } \\
\hline Алоглиптин 25 мг & 7,93 & $\begin{array}{c}-1,10 \\
(-1,38 ;-0,82)\end{array}$ & $\begin{array}{c}56 \% \\
(32-78 \%)\end{array}$ & $\begin{array}{c}0,39 \% \\
(0,028-1,7 \%)\end{array}$ & - & $\begin{array}{c}-0,45 \\
(-2,22 ; 1,31)\end{array}$ \\
\hline Линаглиптин 5 мг & 8,00 & $\begin{array}{c}-0,99 \\
(-1,17 ;-0,82)\end{array}$ & $\begin{array}{c}41 \% \\
(22-63 \%)\end{array}$ & $\begin{array}{c}1,2 \% \\
(0,36-2,8 \%)\end{array}$ & 83,0 & $\begin{array}{c}-0,54 \\
(-6,31 ; 5,09)\end{array}$ \\
\hline Саксаглиптин 5 мг & 8,43 & $\begin{array}{c}-1,03 \\
(-1,21 ;-0,85)\end{array}$ & $\begin{array}{c}31 \% \\
(17-50 \%)\end{array}$ & $\begin{array}{c}1,3 \% \\
(0,45-3,0 \%)\end{array}$ & 81,5 & - \\
\hline Ситаглиптин 100 мг & 8,34 & $\begin{array}{c}-1,06 \\
(-1,22 ;-0,91)\end{array}$ & $\begin{array}{c}38 \% \\
(22-57 \%)\end{array}$ & $\begin{array}{c}2,1 \% \\
(0,74-4,7 \%)\end{array}$ & 83,8 & $\begin{array}{c}-0,99 \\
(-2,38 ; 0,35)\end{array}$ \\
\hline Вилдаглиптин 50 мг x 2 раза & 7,86 & $\begin{array}{c}-1,02 \\
(-1,18 ;-0,86)\end{array}$ & $\begin{array}{c}35 \% \\
(18-54 \%)\end{array}$ & $\begin{array}{c}1,2 \% \\
(0,37-3,1 \%)\end{array}$ & 90,0 & $\begin{array}{c}0,15 \\
(-0,99 ; 1,28)\end{array}$ \\
\hline
\end{tabular}

Примечание: *Средневзвешенное значение.

приема препарата, период полувыведения составляет 36-39 ч. В южнокорейской популяции пациентов с СД2 было проведено исследование по сравнению эффективности и безопасности монотерапии эвоглиптином в дозировках 2,5 мг, 5 мг, 10 мг один раз в день в сравнении с плацебо. Средний уровень гликемии натощак у пациентов был 8,28 ммоль/л, средний $\mathrm{HbA}_{1 с}-7,6 \%$, период терапии эвоглиптином составил 12 нед, после чего оценивались конечные точки. По результатам было продемонстрировано клинически и статистически значимое снижение уровня $\mathrm{HbA}_{1 c}$ на 0,46\%, 0,57\% и 0,51\% по сравнению с плацебо в группах на 2,5, 5 и 10 мг эвоглиптина соответственно; эффективность, зарегистрированная при приеме различных дозировок препарата, статистически не различалась. Препарат не оказывал существенного влияния на массу тела пациентов и значимо улучшал секрецию инсулина [25].

В исследовании Oh и соавт. были оценены эффективность и безопасность эвоглиптина у пациентов с СД2 при различной выраженности почечной дисфункции. Плазменная концентрация эвоглиптина и степень ингибирования активности ДПП-4 увеличивались по мере снижения почечной функции. Средняя область под кривой в период от 0 до 120 ч после однократного приема препарата увеличилась в 1,2, 1,8 и 1,98 раз при легкой, умеренной и тяжелой хронической почечной недостаточности (ХПН) соответственно по сравнению с группой с сохранной функцией почек. Между тем клинически значимых изменений эффективности и безопасности эвоглиптина продемонстрировано не было [26].

\section{ЦЕЛЬ}

Оценить эффективность и безопасность эвоглиптина 5 мг («Донг-А СТ Ко., Лтд», Южная Корея/ООО «ГЕРОФАРМ», Россия) при добавлении к терапии метформином у пациентов с СД2 с неадекватным контролем гликемии. В настоящем исследовании впервые было проведено сравнение эффективности эвоглиптина (5 мг) и ситаглиптина (100 мг) в комбинации с метформином с включением в исследуемую популяцию пациентов из разных этнических групп (Южная Корея и РФ).

\section{МЕТОДЫ}

\section{Дизайн исследования}

Настоящее исследование было спланировано как международное рандомизированное двойное слепое многоцентровое исследование в параллельных группах с активным контролем (2 группы в соотношении 1:1).

\section{Критерии соответствия}

\section{Критерии включения}

В исследование были включены (1) подписавшие информированное добровольное согласие (2) мужчины 


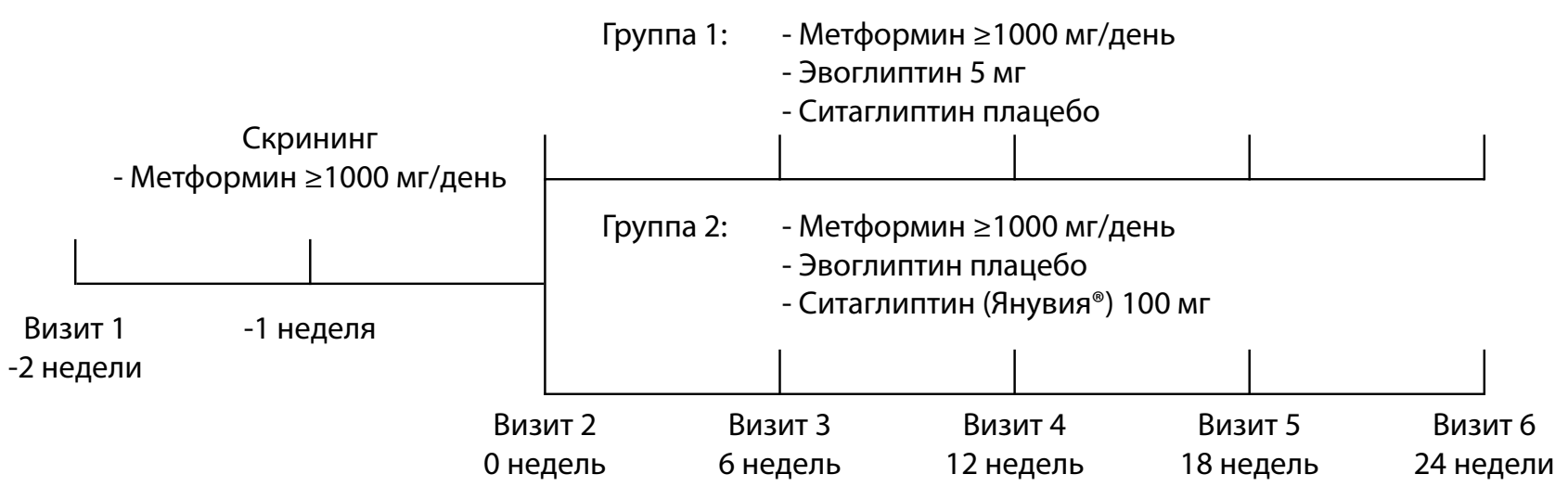

Рис. 1. Схема проведения исследования.

и женщины в возрасте от 18 лет с подтвержденным диагнозом СД2, (3) с уровнем гликированного гемоглобина на скрининге в пределах 6,5-11,0\%, (4) получающие монотерапию метформином в дозе не менее 1000 мг в сутки как минимум в течение 3 мес и последние 6 нед до скрининга, (5) ИМТ которых во время скрининга находился в пределах 20-40 кг/м²; (6) все пациенты подписали согласие придерживаться барьерных методов контрацепции с момента подписания информированного добровольного согласия до окончания участия в исследовании.

\section{Критерии невключения}

(1) Уровень глюкозы в плазме натощак $\geq 15$ ммоль/л на скрининге. (2) СД1, вторичный сахарный диабет, гестационный сахарный диабет. (3) Инфаркт миокарда или острое нарушение мозгового кровообращения (ОНМК) в течение последних 6 мес. (4) Хроническая сердечная недостаточность III или IV класса (по NYHA), цирроз печени, заболевания желчного пузыря, акромегалия, астма, аллергические заболевания кожи, дисфункция щитовидной железы с аномальным уровнем тиреотропного гормона (ТТГ) на скрининге. (5) Коронарное шунтирование, резекция отделов желудочно-кишечного тракта в анамнезе. (6) Показатели аланинаминотрансферазы (АЛТ) и аспартатаминотрансферазы (АСТ), превышающие верхнюю границу нормы более чем в 2,5 раза. (7) Показатель креатинфосфокиназы (КФК), превышающий верхнюю границу нормы более чем в 2,5 раза, с симптомами одышки и боли в груди. (8) Уровень креатинина в сыворотке крови превышающий 132,6 мкмоль/л у мужчин или 123,8 мкмоль/л у женщин). (9) Триглицериды натощак $>$ 4,52 ммоль/л. (10) Пациенты, принимающие в настоящее время оральные контрацептивы, кортикостероиды, варфарин, дигоксин, ингибиторы или индукторы изофермента СYР3А4. (11) Предшествующая терапия инсулином или аналогами глюкагоноподобного пептида 1 (ГПП-1) (в исключительных случаях могли быть включены пациенты, получающие инсулин не более 2 нед в связи с оперативным вмешательством или обследованием) или тиазолидиндионами, или иДПП-4 в течение 6 мес перед скринингом. (12) Анамнестические сведения о злоупотреблении алкоголем или лекарственными препаратами в течение 2 мес до скрининга. (13) Участие в клиническом исследовании препаратов в течение 2 мес до начала исследования. (14) Беременные и кормящие женщины. (15) Любые другие состояния, по обоснованному мнению врача-исследователя, затрудняющие участие пациента в исследовании. (16) Аллергические реакции к компонентам препарата Янувия ${ }^{\circledR}$ (ситаглиптин).

\section{Условия проведения}

Исследование было проведено на базе 33 клинических центров, 6 из которых расположены на территории Российской Федерации, 27 - на территории Южной Кореи.

\section{Продолжительность исследования}

План исследования включал скрининг продолжительностью 2 нед и период лечения, составивший 24 нед. Схема проведения исследования приведена на рис. 1.

\section{Описание медицинского вмешательства}

Все пациенты, соответствовавшие критериям включения и не имевшие критериев невключения, были рандомизированы в две группы с использованием метода стратификационной рандомизации в зависимости от значения гликированного гемоглобина $\mathrm{HbA}_{1 c}$ при скрининге $\left(\mathrm{HbA}_{1 c}<8,5 \%\right.$ или $\left.\mathrm{HbA}_{1 c} \geq 8,5 \%\right)$. На протяжении периода скрининга все пациенты продолжали принимать метформин в той же дозе, в которой принимали препарат до скрининга. В дальнейшем пациенты продолжали принимать метформин, а также начинали прием исследуемого препарата (эвоглиптин, таблетки, покрытые пленочной оболочкой, 5 мг) или препарат сравнения (ситаглиптин, таблетки, покрытые пленочной оболочкой, 100 мг) в сочетании с плацебо. Посещение исследовательского центра было запланировано для пациентов с 6-недельным интервалом, при этом при каждом посещении центра врач-исследователь производил контроль за соблюдением рекомендуемой диеты и физической активности, мониторинг сопутствующей терапии, физикальный осмотр, оценку ИМТ, измерение жизненно важных показателей (артериального давления, частоты сердечных сокращений, частоты дыхательных движений), клинического и биохимического анализа крови, общего анализа мочи, уровня $\mathrm{HbA}_{1 c^{\prime}}$ концентрации глюкозы в плазме натощак и регистрацию нежелательных явлений.

\section{Условия досрочного прекращения терапии}

Пациент мог досрочно прекратить участие в исследовании, если: (1) потребовал прекращения участия в исследовании (отзыв информированного согласия или отказ от посещения клинического центра); (2) принял лекарственный препарат, который, как ожидалось, 
мог повлиять на оценку эффективности и безопасности исследуемого препарата, без назначения главного исследователя; (3) было трудно продолжить участие в исследовании из-за серьезной нежелательной реакции или клинически значимого отклонения в лабораторных анализах, в том числе развития острого панкреатита на фоне лечения. Пациенты также выбывали из исследования, если (4) на фоне резервного лечения (глимепирид) в течение 2 нед содержание глюкозы в крови натощак превышало 15,0, 13,3 и 11,1 ммоль/л после визитов 2, 4 и 5 соответственно; если (5) на фоне лечения у пациента однократно был зарегистрирован эпизод тяжелой или симптоматической гипогликемии или двукратно - эпизоды бессимптомной гипогликемии; если (6) исследователь принял решение о прекращении участия пациента в исследовании.

\section{Основной исход исследования}

Первичной конечной точкой в настоящем исследовании являлось изменение уровня $\mathrm{HbA}_{1 с}$ (\%) на 24-й неделе по сравнению с исходными значениями. При этом для доказательства не меньшей эффективности препарата эвоглиптин по сравнению с препаратом ситаглиптин по данному показателю было необходимо, чтобы верхняя граница 95\% двустороннего доверительного интервала (ДИ) для средней разницы между группами изменения уровня $\mathrm{HbA}_{1 c}$ на 24-й неделе по сравнению с исходными значениями не превышала 0,35\%.

\section{Дополнительные исходы исследования}

Вторичными конечными точками в настоящем исследовании являлись: (1) изменение концентрации глюкозы в плазме натощак (FPG) на 24-й неделе по сравнению с исходным значением; (2) изменение параметров липидного профиля (общий холестерин, липопротеиды Высокой плотности (ЛПВП), липопротеиды низкой плотности (ЛПНП), триглицериды (ТГ), свободные жирные кислоты (СЖК)) на 24-й неделе по сравнению с исходными значениями; (3) доля пациентов, достигших уровня $\mathrm{HbA}_{1 c} \leq 6,5 \%$ на 24-й неделе лечения; (4) количество случаев, требующих проведения немедленной коррекции гипергликемии; (5) изменение уровня базального инсулина и С-пептида на 24-й неделе относительно исходного уровня; (6) изменение индекса инсулинорезистентности HOMA-IR на 24-й неделе относительно исходного уровня; (7) изменение индекса функции $\beta$-клеток поджелудочной железы НОМА- $\beta$ на 24-й неделе относительно исходного уровня; (8) изменение индекса QUICKI на 24-й неделе относительно исходного уровня; (9) изменение массы тела на 24-й неделе по сравнению с исходным значением; (10) изменение показателя среднесуточной глюкозы в крови (MDG) на 24-й неделе по сравнению с исходным значением.

\section{Оценка безопасности}

Критериями оценки безопасности в настоящем исследовании являлись: (1) частота и тяжесть возникновения нежелательных явлений; (2) изменения в показателях физикального осмотра; (3) изменение жизненно важных показателей относительно исходного уровня; (4) изменение инструментальных показателей (ЭКГ в 12 отведениях) относительно исходного уровня; (5) ча- стота возникновения эпизодов гипогликемии (уровень глюкозы в плазме <3,9 ммоль/л); (6) изменения показателей клинического и биохимического анализов крови и общего анализа мочи относительно исходного уровня.

\section{Анализ в подгруппах}

Анализ подгрупп проводился для первичной точки эффективности - изменения уровня $\mathrm{HbA}_{1 с}$ и вторичной точки эффективности - изменения уровня глюкозы в плазме натощак (FPG) на FA-множестве по 5 факторам: уровню $\mathrm{HbA}_{1 c}$ на скрининге $\left(\mathrm{HbA}_{1 c}<8,5 \%\right.$ и 8,5\%), полу, возрасту (до 65 лет, 65 лет и старше на момент скрининга), ИМТ ( $\leq 25,>25$ на момент скрининга), а также по стране проведения исследования (Россия, Южная Корея).

\section{Методы регистрации исходов}

Физикальный осмотр и лабораторно-инструментальное обследование пациентов производились по стандартным протоколам.

Определение среднесуточной глюкозы в крови было проведено по показателям уровня глюкозы в крови, регистрируемым в дневнике самоконтроля пациента. Среднесуточный уровень глюкозы - это среднее значение измерений уровня глюкозы, выполненных 7 раз в день (перед каждым приемом пищи, через 2 ч после каждого приема пищи и перед сном) в один из 7 дней до дня визита с использованием глюкометра. Данные включались в анализ только при наличии всех 7 измерений уровня глюкозы на Неделе 0 и на Неделе 24.

Индекс HOMA-IR рассчитывался по формуле:

$$
\text { HOMA-IR }=\frac{\text { глюкоза натощак }\left(\frac{\text { ммоль }}{\pi}\right) * \text { инсулин натощак }\left(\frac{\text { мкЕд }}{\text { мл }}\right)}{22,5}
$$

Индекс НОМА-В рассчитывался по формуле:

$$
\text { НОМА-B }=\frac{20 \text { * инсулин натощак }\left(\frac{\text { мкЕд }}{\text { мл }}\right)}{\text { глюкоза натощак }\left(\frac{\text { ммоль }}{\pi}\right)-3,5}
$$

Индекс QUICKI рассчитывался по формуле:

$$
\text { НОМА-B }=\frac{1}{\log \left(\text { инсулин натощак }\left(\frac{\text { мкЕД }}{\text { мл }}\right)+\log \left(\text { глюкоза натощак }\left(\frac{\text { ммоль }}{\pi}\right) * 18\right)\right.}
$$

\section{Этическая экспертиза}

В РФ все основные документы настоящего исследования (протокол, брошюра исследователя, информированное согласие, документы по страхованию жизни и здоровья пациентов) были одобрены Советом по этике (Заключение заседания Совета по этике № 118 от 02.02.2016 г.). В Корее разрешение Министерства по безопасности пищевых продуктов и медикаментов (Ministry of Food and Drug Safety) на проведение клинического исследования было получено 11.04.2013 г. (Разрешение № 11953). Все Независимые Этические комитеты (НЭК) исследовательских центров также одобрили проведение исследования.

\section{Статистический анализ}

\section{Принципы расчета размера выборки}

Для расчета размера выборки были проанализированы результаты предыдущих исследований 24-недельного 
Таблица 2. Исходные демографические, антропометрические и анамнестические характеристики пациентов, n(\%)

\begin{tabular}{|c|c|c|c|}
\hline Показатель (среднее \pm станд. откл.) & $\begin{array}{c}\text { Эвоглиптин } \\
(n=142)\end{array}$ & $\begin{array}{c}\text { Ситаглиптин } \\
(n=139)\end{array}$ & p-value \\
\hline Возраст, лет & $57,43 \pm 9,50$ & $57,86 \pm 9,23$ & 0,703 \\
\hline$\leq 34$ лет, n (\%) & $3(2,1)$ & $1(0,7)$ & \\
\hline 35-44 года, n (\%) & $9(6,3)$ & $10(7,2)$ & \\
\hline 45-54 года, n (\%) & $39(27,5)$ & $42(30,2)$ & \\
\hline 55-64 года, n (\%) & $55(38,7)$ & $47(33,8)$ & 0,652 \\
\hline 65-74 года, n (\%) & $34(23,9)$ & $35(25,2)$ & \\
\hline 75-84 года, n (\%) & $1(0,7)$ & $4(2,9)$ & \\
\hline$\geq 85$ лет, n (\%) & $1(0,7)$ & $0(0,0)$ & \\
\hline \multicolumn{4}{|l|}{ Пол } \\
\hline мужской, n (\%) & $65(45,8)$ & $66(47,5)$ & \multirow[t]{2}{*}{0,867} \\
\hline женский, n (\%) & $77(54,2)$ & $73(52,5)$ & \\
\hline Масса тела, кг & $71,55 \pm 15,87$ & $70,87 \pm 14,96$ & 0,880 \\
\hline Рост, сM & $163,51 \pm 8,80$ & $162,94 \pm 9,38$ & 0,432 \\
\hline 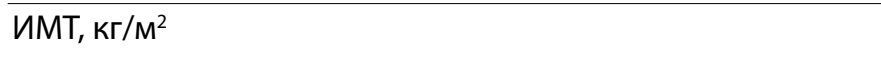 & $26,58 \pm 4,35$ & $26,49 \pm 3,83$ & 0,779 \\
\hline$\leq 25, \mathrm{n}(\%)$ & $59(41,5)$ & $55(39,6)$ & \multirow{2}{*}{0,829} \\
\hline$>25, \mathrm{n}(\%)$ & $83(58,5)$ & $84(60,4)$ & \\
\hline Длительность СД, лет ${ }^{1}$ & $8,59 \pm 5,49$ & $7,74 \pm 4,66$ & 0,260 \\
\hline $\mathrm{HbA}_{1 c}$ на скрининге & $7,45 \pm 0,71$ & $7,46 \pm 0,74$ & 0,734 \\
\hline Ниже 8,5\%, n (\%) & $122(85,9)$ & $123(88,5)$ & \multirow{2}{*}{0,600} \\
\hline 8,5\% и выше, n (\%) & $20(14,1)$ & $16(11,5)$ & \\
\hline Диабетическая ретинопатия, n (\%) & $8(5,8)$ & $14(9,9)$ & 0,245 \\
\hline Диабетическая нейропатия, n (\%) & $25(18,0)$ & $26(18,3)$ & 0,933 \\
\hline Диабетическая нефропатия, n (\%) & $6(4,3)$ & $5(3,5)$ & 1,00 \\
\hline \multicolumn{4}{|c|}{ Нарушения со стороны сердечно-сосудистой системы в анамнезе } \\
\hline Артериальная гипертензия, n (\%) & $58(41,7)$ & $61(43,0)$ & 0,693 \\
\hline Инфаркт миокарда, n (\%) & $3(2,2)$ & $1(0,7)$ & 0,630 \\
\hline ИБС. Стенокардия, n (\%) & $3(2,2)$ & $1(0,7)$ & 0,630 \\
\hline ИБС. Нестабильная стенокардия, n (\%) & $1(0,7)$ & $2(1,4)$ & 0,985 \\
\hline Фибрилляция предсердий, n (\%) & $4(2,9)$ & $1(0,7)$ & 0,380 \\
\hline $\mathrm{XCH}, \mathrm{n}(\%)$ & $1(0,7)$ & $2(1,4)$ & 0,990 \\
\hline
\end{tabular}

Примечания: ' По причине того, что не все участники смогли предоставить точную дату постановки диагноза, длительность диабета была вычислена по формуле: (год скринингового визита - год постановки диагноза + 1). У 5 участников отсутствуют сведения о дате постановки диагноза (для данного показателя в каждой из групп $\mathrm{n}=138$ )

применения препаратов из класса иДПП-4. Расчет необходимого количества пациентов был произведен в следующих предположениях: стандартное отклонение изменения уровня $\mathrm{HbA}_{1 с}$ относительно исходного равно 0,9, граница не меньшей эффективности - 0,35\%, уровень значимости $-2,5 \%$ (а, односторонний), мощность - $80 \%$ (1- $\beta$ ).

\section{Mетоды статистического анализа данных}

Для доказательства не меньшей эффективности препарата эвоглиптин по сравнению с препаратом активного контроля ситаглиптин рассчитывался 95\% двухсторонний ДИ для средней разницы изменения $\mathrm{HbA}_{1 c}$ на 24-й неделе по сравнению с исходным уровнем между группами.
Для анализируемых показателей эффективности и безопасности оценивалась значимость изменений на 24-й неделе относительно исходного уровня внутри каждой группы. В зависимости от типа данных анализ проводился с помощью t-критерия Стьюдента для связанных совокупностей, непараметрического критерия Вилкоксона или непараметрического критерия МаннаУитни. Сравнение между группами в зависимости от типа данных проводилось с помощью t-критерия Стьюдента для независимых групп, непараметрического критерия Манна-Уитни, точного критерия Фишера или критерия $\mathrm{X}^{2}$ Пирсона.

Анализ эффективности по первичной конечной точке проводился на множестве, включающем всех рандоми- 
зированных пациентов, которые начали прием исследуемого препарата и у которых хотя бы один раз оценивался показатель первичной точки эффективности (Full Analysis Set, FA-множество). Для вторичного анализа было выбрано множество пациентов, завершивших исследование, не получавших «терапию спасения» (глимепирид) и не имевших серьезных отклонений от протокола (Per Protocol Set, PP-множество). Оценка безопасности проводилась на множестве пациентов, рандомизированных в исследование и принявших хотя бы одну дозу исследуемого препарата, у которых хотя бы один раз оценивались показатели безопасности.

\section{РЕЗУЛЬТАТЫ}

\section{объекты (участники) исследования}

Для доказательства не меньшей эффективности препарата эвоглиптин по сравнению с препаратом ситаглиптин требовалось в общей сложности 208 пациентов (104 пациента в каждой группе); с учетом $35 \%$ выбывания пациентов из исследования общее число пациентов составило 320 человек (160 пациентов в каждой группе). Всего было скринировано 348 пациентов, не прошли скрининг 67 участников. Рандомизированы в клиническое исследование 281 участник: 142 пациента в группу, получавшую эвоглиптин и метформин (группа 1), и 139 пациентов в группу, получавшую ситаглиптин и метформин (группа 2). Исследование полностью завершили 132 пациента из группы 1 (93,0\%) и 125 пациентов из группы 2 (89,9\%); всего - 257 пациентов. Причинами досрочного прекращения участия в исследовании были «отказ пациента» (8 субъектов), возникновение «нежелательного явления» (8 субъектов), отклонение от протокола исследования (5 субъектов) и «решение исследователя» (3 субъекта). Информация о пациентах, рандомизированных в настоящее исследование, представлена в табл. 2.

Пациенты, включенные в исследование в России, по сравнению с пациентами из Южной Кореи на старте терапии имели более высокий уровень гликемии натощак $(p=0,039)$ и более высокую концентрацию C-пептида в крови $(p=0,002)$. ИМТ у российских пациентов был более высоким по сравнению с южно-корейской субпопуляцией $(p<0,0001)$, и дислипидемия была более выражена ( $p=0,0003$ для концентрации общего холестерина; $p<0,0001$ для концентрации ЛПНП). Субпопуляции не различались по уровню $\mathrm{HbA}_{1 с}$ и индексам инсулинорезистентности на старте терапии. Пациенты в российской субпопуляции имели более высокую скорость клубочковой фильтрации $(p=0,013)$. Артериальная гипертензия чаще была диагностирована у российских пациентов ( $p=0,016)$, которые чаще, чем корейские пациенты, получали терапию ингибиторами АПФ $(p<0,0001)$, при этом контроль артериального давления был удовлетворительным и сопоставимым ( $p=0,316$ для систолического АД и $\mathrm{p}=0,568$ для диастолического АД).

\section{Основные результаты исследования}

Результаты данного исследования представлены впервые.

В анализ эффективности по первичной конечной точке вошли данные 274 пациентов (140 субъектов, прини- мавших эвоглиптин и 134 субъекта, принимавших ситаглиптин). Остальные 7 человек не были включены в Full Analysis Set по причине отсутствия данных о первичной конечной точке.

Изменение $\mathrm{HbA}_{1 c}$ (\%) через 24 нед лечения по сравнению с исходным уровнем составило (среднее \pm станд. откл.) -0,58 \pm 0,70 в группе пациентов, принимавших эвоглиптин, и -0,61 \pm 0,66 в группе пациентов, принимавших ситаглиптин ( $p<0,0001$ - в обеих группах). Различие средних значений между исследуемыми группами в изменении $\mathrm{HbA}_{1 c}$ на 24-й неделе составило 0,03\% [95\% ДИ: -0,14; $0,19 \%]$, что ниже предела не меньшей эффективности 0,35\% и доказывает не меньшую эффективность препарата эвоглиптин по сравнению с препаратом ситаглиптин. Проведенный post-hoc анализ первичной конечной точки с включением принимаемого лекарственного препарата, этнической группы и исследовательского центра в качестве ковариат показал, что и одни из перечисленных ковариат не была статистически значимой ( $>>0,05)$. Изменения уровня $\mathrm{HbA}_{1 c}$ на фоне лечения приведены на рис. 2.

\section{Дополнительные результаты исследования}

Доля пачиентов, достигших уровня $\mathrm{HbA}_{1 c} \leq 6,5 \%$

Результаты анализа в РР-множестве показали, что доля пациентов, уровень $\mathrm{HbA}_{1 с}$ которых был $<6,5 \%$ через 24 нед лечения, составила 31,67\% в группе принимавших эвоглиптин (38 из 120) и 36,61\% - в группе принимавших ситаглиптин (41 из 112); статистически значимое различие между группами отсутствовало $(p=0,345)$.

Количество случаев, требующих проведения немедленной коррекции гипергликемии

Мероприятия по немедленной коррекции гипергликемии и последующее назначение терапии спасения (глимепирид) потребовались 2 пациентам, принимавшим эвоглиптин, и не понадобились никому из принимавших ситаглиптин. Риск гипергликемических состояний не отличался между группами $(p=0,497)$.

\section{Лабораторные показатели}

Исходные значения и изменения параметров пациентов по лабораторным показателям вторичных конечных точек приведены в табл. 3. Анализ производил-

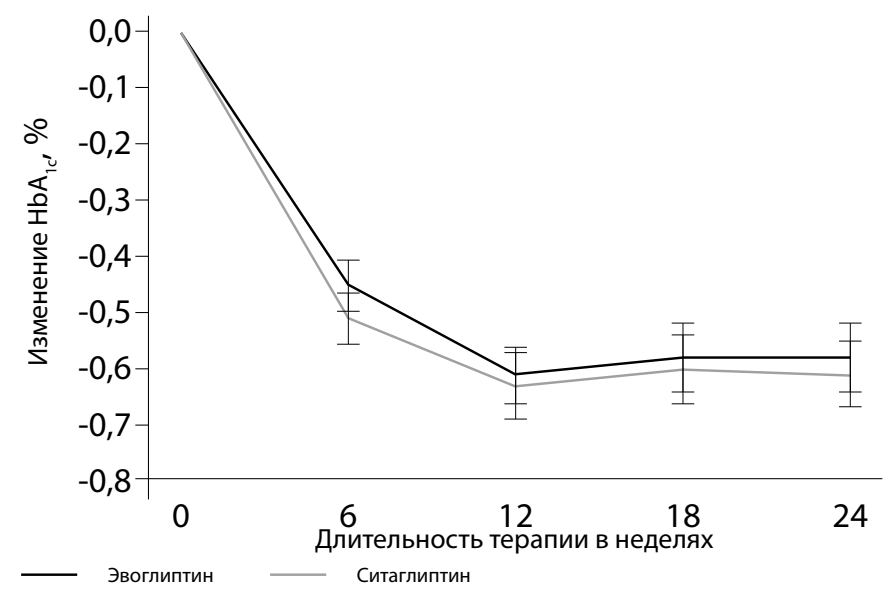

Рис. 2. Изменение $\mathrm{HbA}_{1 с}$ на фоне лечения (среднее \pm стандартная ошибка). 
Таблица 3. Исходные демографические, антропометрические и анамнестические характеристики пациентов

\begin{tabular}{|c|c|c|c|}
\hline $\begin{array}{c}\text { Показатель } \\
\text { (среднее } \pm \text { станд. откл.) }\end{array}$ & $\begin{array}{c}\text { Эвоглиптин } \\
(n=120)\end{array}$ & $\begin{array}{l}\text { Ситаглиптин } \\
(n=112)\end{array}$ & $\begin{array}{c}\text { p-value } \\
\text { (между группами) }\end{array}$ \\
\hline \multicolumn{4}{|l|}{ Уровень гликемии натощак, ммоль/л } \\
\hline Неделя 0 & $7,54 \pm 1,67$ & $7,91 \pm 1,75$ & ns \\
\hline Изменение через 24 нед & $-0,54 \pm 1,55$ & $-0,75 \pm 1,33$ & ns \\
\hline$p$-value & $<0,0001$ & $<0,0001$ & \\
\hline \multicolumn{4}{|l|}{ Уровень инсулина натощак, мкМЕ/мл } \\
\hline Неделя 0 & $8,68 \pm 6,55$ & $8,74 \pm 4,54$ & ns \\
\hline Изменение через 24 нед & $0,83 \pm 5,51$ & $0,50 \pm 3,88$ & ns \\
\hline$p$-value & ns & ns & \\
\hline \multicolumn{4}{|l|}{ Уровень С-пептида натощак, нг/мл } \\
\hline Неделя 0 & $2,30 \pm 1,21$ & $2,40 \pm 1,01$ & ns \\
\hline Изменение через 24 нед & $0,06 \pm 0,86$ & $0,16 \pm 1,02$ & ns \\
\hline p-value & ns & ns & \\
\hline \multicolumn{4}{|l|}{ Индекс НОМА- $\beta, \%$} \\
\hline Неделя 0 & $47,64 \pm 32,25$ & $45,45 \pm 30,45$ & ns \\
\hline Изменение через 24 нед & $11,60 \pm 28,55$ & $13,98 \pm 26,13$ & ns \\
\hline p-value & $<0,0001$ & $<0,0001$ & \\
\hline \multicolumn{4}{|l|}{ Индекс HOMA-IR } \\
\hline Неделя 0 & $2,94 \pm 2,56$ & $3,10 \pm 1,84$ & ns \\
\hline Изменение через 24 нед & $0,10 \pm 2,31$ & $-0,09 \pm 1,62$ & ns \\
\hline p-value & ns & ns & \\
\hline \multicolumn{4}{|l|}{ Индекс QUICKI } \\
\hline Неделя 0 & $0,15 \pm 0,01$ & $0,14 \pm 0,01$ & ns \\
\hline Изменение через 24 нед & $0,00 \pm 0,01$ & $0,00 \pm 0,01$ & ns \\
\hline p-value & ns & ns & \\
\hline \multicolumn{4}{|c|}{ Среднесуточный уровень гликемии, ммоль/л } \\
\hline Неделя 0 & $8,90 \pm 1,92$ & $9,20 \pm 2,00$ & ns \\
\hline Изменение через 24 нед & $-0,95 \pm 1,51$ & $-1,32 \pm 1,56$ & ns \\
\hline$p$-value & $<0,0001$ & $<0,0001$ & \\
\hline \multicolumn{4}{|l|}{ Масса тела, кг } \\
\hline Неделя 0 & $71,26 \pm 15,48$ & $70,55 \pm 15,38$ & ns \\
\hline Изменение через 24 нед & $0,33 \pm 1,98$ & $0,29 \pm 2,18$ & ns \\
\hline p-value & ns & ns & \\
\hline \multicolumn{4}{|l|}{ Общий холестерин, ммоль/л } \\
\hline Неделя 0 & $4,51 \pm 1,09$ & $4,43 \pm 1,07$ & ns \\
\hline Изменение через 24 нед & $-0,10 \pm 0,81$ & $-0,01 \pm 0,64$ & ns \\
\hline$p$-value & ns & ns & \\
\hline \multicolumn{4}{|l|}{ ЛПНП, ммоль/л } \\
\hline Неделя 0 & $2,75 \pm 1,02$ & $2,68 \pm 0,97$ & ns \\
\hline Изменение через 24 нед & $-0,10 \pm 0,67$ & $-0,04 \pm 0,56$ & ns \\
\hline p-value & ns & ns & \\
\hline \multicolumn{4}{|l|}{ ЛПВП, ммоль/л } \\
\hline Неделя 0 & $1,35 \pm 0,29$ & $1,31 \pm 0,35$ & ns \\
\hline Изменение через 24 нед & $0,01 \pm 0,22$ & $0,00 \pm 0,24$ & ns \\
\hline p-value & ns & ns & \\
\hline
\end{tabular}


Таблица 3. Исходные демографические, антропометрические и анамнестические характеристики пациентов (окончание)

\begin{tabular}{|c|c|c|c|}
\hline $\begin{array}{c}\text { Показатель } \\
\text { (среднее } \pm \text { станд. откл.) }\end{array}$ & $\begin{array}{c}\text { Эвоглиптин } \\
(n=120)\end{array}$ & $\begin{array}{c}\text { Ситаглиптин } \\
(n=112)\end{array}$ & $\begin{array}{c}\text { p-value } \\
\text { (между группами) }\end{array}$ \\
\hline \multicolumn{4}{|l|}{ Триглицериды, ммоль/л } \\
\hline Неделя 0 & $1,56 \pm 0,80$ & $1,65 \pm 0,74$ & ns \\
\hline Изменение через 24 нед & $-0,03 \pm 0,82$ & $0,09 \pm 0,85$ & ns \\
\hline$p$-value & ns & ns & \\
\hline \multicolumn{4}{|l|}{ Свободные жирные кислоты, мкЭкв/л ${ }^{1}$} \\
\hline Неделя 0 & $673,86 \pm 252,98$ & $688,21 \pm 278,12$ & ns \\
\hline Изменение через 24 нед & $-25,46 \pm 269,96$ & $-67,85 \pm 276,32$ & ns \\
\hline$p$-value & ns & 0,021 & \\
\hline
\end{tabular}

Примечания: «ns» = «non-significant» - статистически значимых различий не выявлено $(\mathrm{p}>0,05)$

${ }^{1}$ У части пациентов лабораторный анализ СЖК был проведен с определением СЖК методом газовой хроматографии с масс-селективным детектированием (ГХ-МС) с помощью которого были определены точные концентрации насыщенных и мононенасыщенных жирных кислот по отдельности, без определения общего показателя СЖК. В связи с этим добавление результатов не представляется корректным, в таблице представлены результаты определения СЖК в РР-множестве 182 пациентов (95 пациентов группы 1 и 87 пациентов группы 2).

таблица 4. Анализ подгрупп для показателя «изменение $\mathrm{HbA}_{1 с}$ через 24 недели лечения, \%»

\begin{tabular}{lccccc}
\hline \multicolumn{1}{c}{ Подгруппа } & $\mathbf{n}$ & Эвоглиптин & $\mathbf{n}$ & Ситаглиптин & р-value \\
\hline $\mathrm{HbA}_{1 с}<8,5 \%$ & 119 & $-0,47[-0,82 ;-0,14]$ & 118 & $-0,56[-0,90 ;-0,20]$ & 0,322 \\
$\mathrm{HbA}_{1 с} \geq 8,5 \%$ & 19 & $-1,21[-1,78 ;-0,56]$ & 16 & $-1,34[-1,88 ;-1,02]$ & 0,679 \\
Мужчины & 63 & $-0,60[-1,02 ;-0,19]$ & 64 & $-0,69[-1,11 ;-0,30]$ & 0,558 \\
Женщины & 75 & $-0,47[-0,82 ;-0,14]$ & 70 & $-0,56[-0,82 ;-0,21]$ & 0,481 \\
До 65 лет & 103 & $-0,52[-0,91 ;-0,23]$ & 97 & $-0,66[-1,00 ;-0,20]$ & 0,643 \\
65 лет и старше & 35 & $-0,44[-0,77 ;-0,10]$ & 37 & $-0,56[-0,83 ;-0,37]$ & 0,168 \\
ИМт <25 кг/м ${ }^{2}$ & 59 & $-0,52[-0,90 ;-0,21]$ & 54 & $-0,56[-1,02 ;-0,24]$ & 0,573 \\
ИМт $\geq 25$ кг/м ${ }^{2}$ & 79 & $-0,47[-0,93 ;-0,12]$ & 80 & $-0,64[-0,93 ;-0,27]$ & 0,460 \\
Россия & 27 & $-0,50[-1,00 ;-0,05]$ & 28 & $-0,45[-1,02 ; 0,05]$ & 0,711 \\
Южная Корея & 111 & $-0,52[-0,90 ;-0,18]$ & 106 & $-0,64[-0,97 ;-0,34]$ & 0,702 \\
\hline
\end{tabular}

Таблица 5. Анализ подгрупп для показателя «изменение уровня гликемии натощак через 24 недели лечения, ммоль/л»

\begin{tabular}{lccccc}
\hline \multicolumn{1}{c}{ Подгруппа } & $\mathbf{n}$ & Эвоглиптин & $\mathbf{n}$ & Ситаглиптин & р-value \\
\hline $\mathrm{HbA}_{1 с}<8,5 \%$ & 119 & $-0,33[-1,12 ; 0,16]$ & 118 & $-0,50[-1,19 ; 0,10]$ & 0,387 \\
$\mathrm{HbA}_{1 с} \geq 8,5 \%$ & 19 & $-0,78[-2,02 ; 1,06]$ & 16 & $-1,08[-2,21 ;-0,61]$ & 0,707 \\
Мужчины & 63 & $-0,72[-1,45 ; 0,04]$ & 64 & $-0,53[-1,27 ; 0,00]$ & 0,619 \\
Женщины & 75 & $-0,16[-0,78 ; 0,33]$ & 70 & $-0,56[-1,19 ; 0,28]$ & 0,075 \\
До 65 лет & 103 & $-0,50[-1,32 ; 0,14]$ & 97 & $-0,56[-1,27 ; 0,05]$ & 0,717 \\
65 лет и старше & 35 & $-0,19[-0,72 ; 0,27]$ & 37 & $-0,61[-1,33 ; 0,06]$ & 0,098 \\
ИМТ <25 кг/м ${ }^{2}$ & 59 & $-0,39[-1,14 ; 0,08]$ & 54 & $-0,89[-1,39 ; 0,00]$ & 0,126 \\
ИМт $\geq 25$ кг/м ${ }^{2}$ & 79 & $-0,33[-1,26 ; 0,21]$ & 80 & $-0,44[-1,17 ; 0,17]$ & 0,738 \\
Россия & 27 & $0,07[-0,94 ; 1,06]$ & 28 & $-0,69[-1,22 ; 0,33]$ & 0,104 \\
Южная Корея & 111 & $-0,50[-1,25 ; 0,06]$ & 106 & $-0,50[-1,28 ; 0,06]$ & 0,702 \\
\hline
\end{tabular}

ся на РР-множестве пациентов. В обеих группах было зарегистрировано статистически значимое снижение уровня гликемии натощак и среднесуточной гликемии и возрастание индекса НОМА- $\beta$ через 24 нед лечения ( $p<0,0001)$. У пациентов, получавших ситаглиптин, было также зарегистрировано снижение общей концентрации свободных жирных кислот ( $p=0,021)$. Изменение прочих показателей не являлось статистически значимым. Группы не различались ни по одной из вторичных конечных точек.

\section{Анализ подгрупп}

При проведении анализа подгрупп не было выявлено статистически значимых различий в эффективности эвоглиптина и ситаглиптина ни в одной из подгрупп. Результаты анализа подгрупп (медиана, доверительный интервал) приведены в табл. 4 для показателя изменения $\mathrm{HbA}_{1 с}$ и в табл. 5 - для показателя изменения гликемии натощак.

С учетом ранее опубликованных данных о большей эффективности иДПП-4 у представителей монголоидной 
Таблица 6. Сведения о наиболее частых нежелательных явлениях и нежелательных лекарственных реакциях, возникших при проведении исследования

\section{Событие}

\section{Всего HЯ}

Всего СНЯ

Нарушения со стороны ЖКТ

Инфекционные и паразитарные заболевания

Нарушения со стороны скелетно-мышечной и соединительной ткани

Нарушения со стороны кожи и подкожной ткани

Всего НЛР

Нарушения со стороны ЖКТ

Нарушения со стороны кожи и подкожных тканей

Эвоглипти
(140 пациенто
$61(43,6)$
$5(3,6)$
$17(12,1)$
$16(11,4)$
$8(5,7)$
$8(5,7)$
$24(17,1)$
$5(3,6)$
$5(3,6)$

\section{Ситаглиптин,} (136 пациентов) $n$ (\%)

\begin{tabular}{cc}
$51(37,5)$ & $\mathrm{ns}$ \\
$6(4,4)$ & $\mathrm{ns}$ \\
$11(8,1)$ & $\mathrm{ns}$ \\
$15(11,0)$ & $\mathrm{ns}$ \\
$10(7,4)$ & $\mathrm{ns}$ \\
$<5 \%$ & $\mathrm{~ns}$ \\
$7(5,1)$ & 0,015 \\
$3(2,2)$ & $\mathrm{ns}$ \\
$2(1,5)$ & $\mathrm{ns}$ \\
\hline
\end{tabular}

Примечания: «ns» = «non-significant» - статистически значимых различий не выявлено $(p>0,05)$.

НЯ - нежелательное явление - любое выявленное у субъекта клинического исследования после применения лекарственного продукта неблагоприятное с медицинской точки зрения медицинское событие, которое может и не иметь причинно-следственной связи с его применением.

СНЯ - серьезное нежелательное явление - любое НЯ, которое вне зависимости от дозы лекарственного продукта привело к смерти, представляло собой угрозу для жизни, потребовало госпитализации или ее продления, привело к стойкой или значительной нетрудоспособности или инвалидности, представляло собой врожденную аномалию или дефект рождения.

НЛР - нежелательная лекарственная реакция - НЯ, для которого нельзя исключить наличие причинно-следственной связи с приемом лекарственного препарата.

ЖКТ - желудочно-кишечный тракт.

расы [17], дополнительно было проведено сравнение эффективности эвоглиптина в российской и южнокорейской субпопуляциях. При сравнении субпопуляций по вторичным конечным точкам было получено несколько большее снижение уровня гликемии натощак в южнокорейской субпопуляции $(p=0,030)$ при сопоставимом снижении $\mathrm{HbA}_{1 c}(\mathrm{p}=0,657)$. Аналогичные результаты были получены и при сравнении эффективности ситаглиптина в субпопуляциях, что соответствует ранее опубликованным данным.

\section{Нежелательные явления}

При проведении анализа безопасности раздельно оценивали частоту возникновения нежелательных явлений (НЯ) и нежелательных лекарственных реакций (НЛР). Ни одно из серьезных нежелательных явлений (СНЯ) не было определено как возникшее вследствие применения исследуемого препарата.

В группе пациентов, принимавших эвоглиптин, было зарегистрировано статистически значимо больше НЛР $(p=0,015)$, но при этом большинство из них были легкой степени. Было отмечено только 2 случая средней степени тяжести (1 случай в группе пациентов, принимающих эвоглиптин, и 1 случай в группе пациентов, принимающих ситаглиптин). Тяжелых НЛР не отмечалось в обеих группах. Серьезных НЛР выявлено не было. В ходе сравнения между группами пациентов частоты возникновения НЛР не было выявлено статистически значимых различий. Сведения о частоте НЯ, СНЯ, НЛР и наиболее частых НЯ и НЛР приведены в табл. 6.

За время проведения исследования при приеме эвоглиптина был зарегистрирован 1 эпизод стенокардии (степень тяжести - легкое НЯ), 1 эпизод нестабильной стенокардии (тяжелое НЯ) и 1 случай развития острого инфаркта миокарда (тяжелое НЯ). Связь с приемом пре- парата отсутствовала во всех трех эпизодах. При приеме ситаглиптина у 1 пациента было зарегистрировано нарушение ритма сердца (легкое НЯ) и у 1 пациента - полная атриовентрикулярная блокада (тяжелое НЯ). Связь с приемом препарата в обоих случаях также отсутствовала.

Поскольку нарушения со стороны желудочно-кишечного тракта являются в некоторой мере ожидаемым НЯ при приеме инкретиномиметиков, дополнительно был проведен сравнительный анализ частоты возникновения НЯ этой группы в российской и южнокорейской субпопуляциях. Процент пациентов, у которых было зарегистрировано какое-либо НЯ со стороны желудочно-кишечного тракта (диспепсия, диарея, констипация, тошнота, рвота, дискомфорт в эпигастральной области и другие), был ниже в российской субпопуляции как при приеме эвоглиптина (4\% и $14 \%$ соответственно, $\mathrm{p}=0,014$ ), так и при приеме ситаглиптина (3\% и 9\% соответственно, $p=0,014$ ).

Отдельно производился анализ частоты возникновения гипогликемических эпизодов. Между группами лечения не было выявлено статистически значимых различий в частоте возникновения гипогликемии $(p=0,365)$ и ее типов: точно установленной симптоматической гипогликемии $(p=0,242)$, бессимптомной гипогликемии $(p=0,618)$, вероятной симптоматической гипогликемии $(p=0,493)$. Всего были зарегистрированы 1 гипогликемический эпизод в группе эвоглиптина (бессимптомная гипогликемия) и 7 эпизодов - в группе ситаглиптина (3 точно установленных симптоматических гипогликемии, 1 вероятно симптоматическая гипогликемия, 3 бессимптомных гипогликемии). Все эпизоды гипогликемии были зарегистрированы только у пациентов из Южной Кореи. Эпизодов тяжелой гипогликемии за время исследования не наблюдалось. 


\section{ОБСУЖДЕНИЕ}

\section{Резюме основного результата исследования}

Результатом рандомизированного с активным контролем двойного слепого исследования III фазы стало получение доказательств не меньшей эффективности нового высокоселективного иДПП-4 эвоглиптина по сравнению с эталонным препаратом ситаглиптином. Важными особенностями этого исследования было включение пациентов различных этнических групп. Как было указано ранее, в литературе имеются данные, продемонстрировавшие большую эффективность иДПП-4 в азиатской популяции [17], что подтвердилось по результатам анализа подгрупп в настоящем исследовании (не исключена роль конфаундинга различий ИМТ на старте терапии). В то же время при сравнении эффективности эвоглиптина и ситаглиптина внутри российской и корейской субпопуляций в отдельности была продемонстрирована равная эффективность эвоглиптина, не меньшая по сравнению с эффективностью ситаглиптина.

\section{Обсуждение основного результата исследования}

В исследовании было продемонстрировано улучшение $\beta$-клеточной функции, оцененной по индексу НОМА- $\beta$, сопоставимое в обеих группах. Параметры, оценивающие инсулинорезистентность (уровень инсулина и C-пептида натощак, индекс HOMA-IR и индекс QUICKI), в обеих группах остались без изменения, как и масса тела пациентов. Данный результат ожидаем, учитывая, что основной точкой приложения иДПП-4 является секреторная функция эндокриноцитов ( $а-$ и $\beta$-клеток) поджелудочной железы.

Эффекты на другие метаболические параметры - уровень липидов в плазме крови и уровень АД были статистически не значимы и сопоставимы в обеих группах. Единственным отличием было снижение уровня СЖК на терапии ситаглиптином. Повышение уровня СЖК в циркуляции часто выявляется у пациентов с ожирением и может участвовать в формировании периферической (мышечной) инсулинорезистентности [27]. Есть мнение, что их снижение может улучшать чувствительность тканей к инсулину. Между тем, существенных отличий в динамике параметров инсулинорезистентности в данном исследовании выявлено не было. Для уточнения клинической роли изменения концентрации СЖК на фоне терапии иДПП-4 необходимы дополнительные исследования с длительным периодом наблюдения.

В настоящем исследовании также был проведен сравнительный анализ подгрупп с распределением пациентов на страты в соответствии с уже известными предикторами эффективности препаратов группы иДПП-4 (исходный уровень $\mathrm{HbA}_{1 c^{\prime}}$ пол пациента, возраст, ИМТ, этническая принадлежность), но статистически значимые различия были только при стратификации по исходному уровню $\mathrm{HbA}_{1 \mathrm{c}}$.

Сравнение переносимости и безопасности показало сопоставимые результаты в группах исследования. Общее количество нежелательных явлений было несколько выше в группе эвоглиптина, однако все они были легкими. СНЯ не было зарегистрировано ни в одной из групп. Также не было существенных различий по частоте гипогликемий.

\section{ЗАКЛЮЧЕНИЕ}

В настоящем исследовании продемонстрированы не меньшая эффективность и сопоставимая безопасность препарата эвоглиптин в дозе 5 мг один раз в день по сравнению с ситаглиптином 100 мг один раз в день.

\section{ДОПОЛНИТЕЛЬНАЯ ИНФОРМАЦИЯ}

Источник финансирования. Настоящий анализ выполнен в рамках выполнения работ по гранту Российского научного фонда (проект № 17-75-30052) и софинансирования, предоставленного по данному проекту ООО «Герофарм».

Конфликт интересов. Макаренко И.Е. и Леушева В.В. являются сотрудниками ООО «Герофарм».

Бабенко А.Ю., Мосикян А.А., Шляхто Е.В. декларируют отсутствие явных и потенциальных конфликтов интересов, связанных с публикацией настоящей статьи.

Участие авторов. Бабенко А.Ю. - координирующий исследователь двойного слепого рандомизированного клинического испытания по сравнению эффективности и безопасности эвоглиптина 5 мг и ситаглиптина 100 мг, главный исследователь в ФГБУ «НМИЦ им. В.А. Алмазова». Корректура и рецензирование первичного текста рукописи; Мосикян А.А. - статистический анализ предоставленных данных (анализ подгрупп), написание первичного текста рукописи; Леушева В.В. - статистический анализ результатов исследования, подготовка статистического отчета; Макаренко И.Е. - обеспечение проведения двойного слепого рандомизированного клинического испытания по сравнению эффективности и безопасности эвоглиптина 5 мг и ситаглиптина 100 мг, подготовка протокола исследования, контроль его проведения в центрах, корректура и рецензирование первичного текста рукописи; Шляхто Е.В. - корректура и рецензирование всех версий рукописи. Все авторы внесли существенный вклад в проведение исследования и подготовку статьи, прочли и одобрили финальную версию перед публикацией.

Благодарности. Авторы выражают благодарность исследователям и исследовательским центрам, принимавших участие в исследовании ЭВОКОМБИ.

На территории Российской Федерации:

1. ГБУЗ города Москвы «Эндокринологический диспансер Департамента здравоохранения города Москвы». Главный исследователь Анциферов Михаил Борисович, д.м.н., профессор; главный врач ГБУЗ «Эндокринологический диспансер ДЗМ»; профессор ФГАОУ ВО Первый Московский государственный медицинский университет им. И.М. Сеченова Минздрава России.

2. СПб ГБУз «Городская больница Святой преподобномученицы Елизаветы» (Елизаветинская больница). Главный исследователь Ворохобина Наталья Владимировна, д.м.н., профессор; научный консультант эндокринологического отделения СПбГУз «Елизаветинская больница»; заведующая кафедрой эндокринологии, профессор ГБОУ ВПО Северо-Западный государственный медицинский университет им. И.И. Мечникова.

3. ФГБУ Национальный медицинский исследовательский центр им. В.А. Алмазова Министерства здравоохранения Российской Федерации. Главный исследователь - Бабенко Алина Юрьевна, д.м.н., доцент кафедры внутренних болезней института послевузовского образования СЗФМИЦ, заведующая НИЛ диабетологии института эндокринологии НМИЦ, заместитель директора института эндокринологии по учебной работе.

4. ФГБУ Национальный медицинский исследовательский эндокринологии Минздрава России. Главный исследователь - Майоров Александр Юрьевич, д.м.н., заведующий отделом прогнозирования и инноваций диабета. 
5. Филиал негосударственного частного учреждения здравоохранения «Научный клинический центр» Открытого акционерного общества «Российские железные дороги». Главный исследователь - Войчик Эмма Анатольевна, к.м.н., руководитель эндокринологического центра НУЗ «НКЦ ОАО «РЖД».

6. ФГАОУ ВО Первый Московский государственный медицинский университет им. И.М. Сеченова Минздрава России. Главный исследователь - Петунина Нина Александровна, д.м.н., профессор, заведующая кафедрой эндокринологии ФГАОУ ВО Первый МГМУ им. И.М. Сеченова Минздрава России.

На территории Южной Кореи:

7. Больница Канбук Самсунг (Kangbuk Samsung Hospital). Главный исследователь - Сунг-Ву Пак, профессор отделения эндокринологии больницы Кангбук Самсунг (Prof. Sung-Woo Park, Department of Endocrinology, Kangbuk Samsung Hospital).

8. Больница Пресвятого Сердца университета Халлим Кандонга (Hallym University Gangdong Sacred Heart Hospital). Главный исследователь - Ду Ман Ким, профессор отделения эндокринологии и метаболизма больницы Святого Сердца Кандонгу университета Халлим (Prof. Doo Man Kim, Department of Endocrinology and Metabolism, Hallym University Gangdong Sacred Heart Hospital).

9. Медицинский центр Гиль Университета Гачон (Gachon University Gil Medical Center). Главный исследователь - Ки Ёнг Ли, доцент отделения эндокринологии и метаболизма Медицинского центра Гиль Университета Гачон (Assistant Prof. Ki Young Lee, Department of Endocrinology and Metabolism, Gachon University Gil Medical Center).

10. Католический университет Кореи, больница Святой Марии в Сеуле (The Catholic University of Korea Seoul St. Mary's Hospital). Главный исследователь - Кун Хо Юн, профессор отделения эндокринологии и метаболизма больницы Св. Марии Католического Университета Сеула (Prof. Kun Ho Yoon, Catholic University Seoul St. Mary's Hospital).

11. Католический университет Кореи, больница Святой Марии в Пучхоне (Catholic University of Korea Bucheon St. Mary's Hospital). Главный исследователь - Сунг Рэ Ким, доцент отделения эндокринологии и метаболизма больницы Св. Марии Католического университета Бучон (Associate Prof. Sung Rae Kim, Catholic University Bucheon St. Mary's Hospital).

12. Католический университет Кореи, больница Святой Марии в Йеуидо (The Catholic University of Korea Yeouido St. Mary's Hospital). Главный исследователь - Ки Хо Сонг, профессор отделения эндокринологии и метаболизма (Prof. Ki Ho Song, Department of Endocrinology and Metabolism, Catholic University Yeouido St. Mary's Hospital).

13. Больница университета Кёнхи в Кандонге (Kyung Hee University Hospital at Gangdong). Главный исследователь - Кён Чын Ан, профессор отделения эндокринологии больницы университета Кёнхи в Кандонге (Prof. Kyu Jeung Ahn, Department of Endocrinology Gangdong Kyung Hee University Hospital).

14. Больница Кёнбукского национального университета (Kyungpook National University Hospital). Главный исследователь - Ин Кё Ли, профессор отделения эндокринологии больницы Кенбукского национального университета (Prof. In Kyu Lee, Kyungpook National University Hospital).

15. Больница Куро Корейского университета (Korea University Guro Hospital). Главный исследователь - Кунг Мук Чой, профессор отделения эндокринологии и метаболизма больницы Куро Корейского университета (Prof. Kyung Mook Choi, Department of Endocrinology and Metabolism, Korea University Guro Hospital).

16. Больница Мёнджи (Myongji Hospital). Главный исследователь - Джэ Хёк Ли, профессор отделения эндокринологии больницы Мёнгжи (Prof. Jae Hyuck Lee, Department of Endocrinology, Myong Ji Hospital.

17. Медицинский центр Самсунг (Samsung Medical Center). Главный исследователь - Мун Кё Ли, профессор отделения эндокринологии и метаболизма Медицинского центра Самсунг (Prof. Moon Kyu Lee, Department of Endocrinology, Samsung Medical Hospital).

18. Больница Сеульского национального университета (Seoul National University Hospital). Главный исследователь - Кён Су Пак, профессор отделения эндокринологии, метаболизма и внутренних болезней больницы Сеульского университета (Prof. Kyong Soo Park, Department of Endocrinology, Metabolism and Internal Disease, Seoul National University Hospital).

19. Медицинский центр Асан (Asan Medical Center). Главный исследователь - Чун Йол Пак, профессор отделения эндокринологии и метаболизма Медицинского центра Асан (Prof. Joong Yeol Park, Department of Endocrinology and Metabolism, Asan Medical Center).

20. Больница университета Сун Чон Хён, Пучхон (Soon Chun Hyang University Hospital Bucheon). Главный исследователь - Чи О Мок, профессор отделения эндокринологии Сун Чун Хён больницы университета Пучхон. (Prof. Ji Oh Mok, Department of Endocrinology, Bucheon Soonchunhyang University Bucheon Hospital).

21. Больница Северанс, учреждение здравоохранения при университете Йонсей (Severance Hospital, Yonsei University Health System). Главный исследователь - Бон Су Чха, профессор отделения эндокринологии, больница Северанс, учреждение здравоохранения при университете Йонсей (Prof. Bong Soo Cha, Department of Endocrinology, Yonsei University Severance Hospital).

22. Христианская больница Вонджу Северанс (Wonju Severance Christian Hospital). Главный исследователь - Профессор Чхун Хи Чун, Отделение эндокринологии, Христианская больница Вонджу Северанс (Prof. Choon Hee Chung, Department of Endocrinology, Wonju Severance Christian Hospital).

23. Больница общего типа Ыльчи (Eulji General Hospital). Главный исследователь - Кён А Хан, профессор отделения эндокринологии, больница общего типа Ыльчи (Prof. Kyung Ah Han, Department of Endocrinology, Eulji General Hospital).

24. Университет Индже, больница Пусан Пэк (Inje University Busan Paik Hospital). Главный исследователь - Чон Хён Пак, профессор отделения эндокринологии и лечения внутренних болезней, университет Индже больница Пусан Бек (Prof. Jeong Hyun Park, Department of Endocrinology, Internal Medicine, Inje University Busan Paik Hospital).

25. Больница университета Инха (Inha University Hospital). Главный исследователь - Сон Бин Хон, доцент отделения эндокринологии и метаболизма, больница университета Инха (Associate Prof. Seong Bin Hong, Department of Endocrinology and Metabolism, Inha University Hospital).

26. Больница Национального университета Чоннам (Chonnam National University Hospital). Главный исследователь - Мин Ян Чун, профессор отделения эндокринологии и метаболизма, больница Национального университета Чоннам (Prof. Min Young Chung, Department of Endocrinology and Metabolism, Chonnam National University Hospital).

27. Больница национального университета Чеджудо (Jејu National Universiy Hospital). Главный исследователь - Гван Пё Ко, доцент отделения эндокринологии и метаболизма, больница национального университета Чеджу (Prof. Gwan Pyo Koh, Department of Endocrinology and Metabolism, Jeju National University Hospital).

28. Больница Пресвятого Сердца университета Халлим (Hallym University Sacred Heart Hospital). Главный исследователь - Чон Гу Кан, доцент отделение эндокринологии и метаболизма, больница Пресвятого Сердца университета Халлим (Associate Prof. Jun Goo Kang, Department of Endocrinology and Metabolism, Hallym University Sacred Heart Hospital).

29. Больница Кури университета Ханъян (Hanyang University Guri Hospital). Главный исследователь - Чан Пом Ли, профессор отделения эндокринологии и метаболизма, больница Кури университета 
Ханъян (Prof. Chang Beom Lee, Department of Endocrinology and Metabolism, Hanyang University Guri Hospital).

30. Больница Мокдон женского университета Ихва (Ewha Womans University Mokdong Hospital). Главный исследователь - Ён А Сун, профессор отделения эндокринологии, больница Мокдон, женский университет Ихва (Prof. Yeon Ah Sung, Division of Endocrinology, Ewha Womans University Mokdong Hospita).

31. Университетская больница Конянг (Konyang University Hosptial). Главный исследователь - Кён Ён Пак, профессор отделения эндокринологии, университетская больница Конянг (Prof. Keun Yong Park, Department of Endocrinology, Konyang University Hospital).
32. Больница Пундан Сеульского национального университета (Seoul National University Bundang Hospital). Главный исследователь - Су Лим, доцент отделения эндокринологии, больница Пундан, Сеульский национальный университет (Associate Prof. Su Lim, Department of Endocrinology, Seoul National University Bundang Hospital).

33. Медицинский центр Йоннамского университета (Yeungnam University Medical Center). Главный исследователь - Хён Ву Ли, профессор отделения эндокринологии и метаболизма, Медицинский центр Йоннамского университета (Prof. Hyung Woo Lee, Department of Endocrinology and Metabolism, Yongnam University Hospital).

\section{СПИСОК ЛИТЕРАТУРЫ | REFERENCES}

1. Rena G, Hardie DG, Pearson ER. The mechanisms of action of metformin. Diabetologia. 2017;60(9):1577-1585. doi: 10.1007/s00125-017-4342-z

2. Mulvihill EE, Drucker DJ. Pharmacology, physiology, and mechanisms of action of dipeptidyl peptidase-4 inhibitors. Endocr Rev. 2014;35(6):992-1019. doi: 10.1210/er.2014-1035

3. Aschner P, Kipnes MS, Lunceford JK, et al. Effect of the dipeptidyl peptidase-4 inhibitor sitagliptin as monotherapy on glycemic control in patients with type 2 diabetes. Diabetes Care. 2006;29(12):2632-2637. doi: 10.2337/dc06-0703

4. Frederich R, McNeill R, Berglind N, et al. The efficacy and safety of the dipeptidyl peptidase-4 inhibitor saxagliptin in treatment-naive patients with type 2 diabetes mellitus: a randomized controlled trial. Diabetol Metab Syndr. 2012;4(1):36. doi: 10.1186/1758-5996-4-36

5. Pi-Sunyer FX, Schweizer A, Mills D, Dejager S. Efficacy and tolerability of vildagliptin monotherapy in drug-naive patients with type 2 diabetes. Diabetes Res Clin Pract. 2007;76(1):132-138. doi: 10.1016/j.diabres.2006.12.009

6. Barnett AH, Patel S, Harper R, et al. Linagliptin monotherapy in type 2 diabetes patients for whom metformin is inappropriate: an 18 week randomized, double-blind, placebo-controlled phase III trial with a 34-week active-controlled extension. Diabetes Obes Metab. 2012;14(12):1145-1154. doi: 10.1111/dom.12011

7. DeFronzo RA, Fleck PR, Wilson CA, et al. Efficacy and safety of the dipeptidyl peptidase-4 inhibitor alogliptin in patients with type 2 diabetes and inadequate glycemic control: a randomized, double-blind, placebo-controlled study. Diabetes Care. 2008;31(12):23152317. doi: $10.2337 / \mathrm{dc} 08-1035$

8. Derosa G, D'Angelo A, Maffioli P. Sitagliptin in type 2 diabetes mellitus: Efficacy after five years of therapy. Pharmacol Res. 2015;100:127134. doi: 10.1016/j.phrs.2015.07.019

9. Sjostrand M, Wei C, Cook W, et al. Assessment of Saxagliptin Efficacy: Meta-Analysis of 14 Phase 2 and 3 Clinical Trials. Diabetes Ther. 2017;8(3):587-599. doi: 10.1007/s13300-017-0261-8

10. Odawara M, Sagara R. Effects of vildagliptin as add-on treatment in patients with type 2 diabetes mellitus: insights from long-term clinical studies in Japan. J Diabetes Metab Disord. 2015;15:21. doi: 10.1186/s40200-016-0240-z

11. Tinahones FJ, Gallwitz B, Nordaby M, et al. Linagliptin as add-on to empagliflozin and metformin in patients with type 2 diabetes: Two 24-week randomized, double-blind, double-dummy, parallel-group trials. Diabetes Obes Metab. 2017;19(2):266-274. doi: $10.1111 /$ dom.12814

12. Holland DQ, Neumiller JJ. Alogliptin in combination with metformin and pioglitazone for the treatment of type 2 diabetes mellitus. Diabetes Metab Syndr Obes. 2014;7:277-288. doi: 10.2147/DMSO.S37648

13. Esposito K, Cozzolino D, Bellastella G, et al. Dipeptidyl peptidase-4 inhibitors and $\mathrm{HbA} 1 \mathrm{c}$ target of $<7 \%$ in type 2 diabetes: meta-analysis of randomized controlled trials. Diabetes Obes Metab. 2011;13(7):594-603. doi: 10.1111/j.1463-1326.2011.01380.x

14. Esposito K, Chiodini P, Maiorino Ml, et al. A nomogram to estimate the $\mathrm{HbA} 1 \mathrm{c}$ response to different DPP-4 inhibitors in type 2 diabetes: a systematic review and meta-analysis of 98 trials with 24163 patients. BMJ Open. 2015;5(2):e005892. doi: 10.1136/bmjopen-2014-005892
15. Craddy P, Palin HJ, Johnson Kl. Comparative effectiveness of dipeptidylpeptidase-4 inhibitors in type 2 diabetes: a systematic review and mixed treatment comparison. Diabetes Ther. 2014;5(1):1-41. doi: 10.1007/s13300-014-0061-3

16. Yagi S, Aihara K, Akaike M, et al. Predictive Factors for Efficacy of Dipeptidyl Peptidase-4 Inhibitors in Patients with Type 2 Diabetes Mellitus. Diabetes Metab J. 2015;39(4):342-347. doi: 10.4093/dmj.2015.39.4.342

17. Kim YG, Hahn S, Oh TJ, et al. Differences in the glucose-lowering efficacy of dipeptidyl peptidase-4 inhibitors between Asians and non-Asians: a systematic review and meta-analysis. Diabetologia. 2013;56(4):696-708. doi: 10.1007/s00125-012-2827-3

18. Maeda H, Kubota A, Tanaka Y, et al. The safety, efficacy and predictors for $\mathrm{HbA} 1 \mathrm{c}$ reduction of sitagliptin in the treatment of Japanese type 2 diabetes. Diabetes Res Clin Pract. 2012;95(1):e20-22. doi: 10.1016/j.diabres.2011.10.011

19. Pratley RE, McCall T, Fleck PR, et al. Alogliptin use in elderly people: a pooled analysis from phase 2 and 3 studies. J Am Geriatr Soc. 2009;57(11):2011-2019. doi: 10.1111/j.1532-5415.2009.02484.x

20. Mamza J, Mehta R, Donnelly R, Idris I. Determinants of Glycemic Response to Add-On Therapy with a Dipeptidyl Peptidase-4 Inhibitor: A Retrospective Cohort Study Using a United Kingdom Primary Care Database. Diabetes Technol Ther. 2016;18(2):85-92. doi: 10.1089/dia.2015.0052

21. Kim HM, Lim JS, Lee BW, et al. Optimal candidates for the switch from glimepiride to sitagliptin to reduce hypoglycemia in patients with type 2 diabetes mellitus. Endocrinol Metab (Seoul). 2015;30(1):84-91. doi: 10.3803/EnM.2015.30.1.84

22. Scheen AJ, Charpentier G, Ostgren CJ, et al. Efficacy and safety of saxagliptin in combination with metformin compared with sitagliptin in combination with metformin in adult patients with type 2 diabetes mellitus. Diabetes Metab Res Rev. 2010;26(7):540-549. doi: $10.1002 /$ dmrr.1114

23. Goke R, Eschenbach P, Dutting ED. Efficacy of vildagliptin and sitagliptin in lowering fasting plasma glucose: Results of a randomized controlled trial. Diabetes Metab. 2015;41(3):244-247. doi: 10.1016/.jiabet.2014.07.004

24. Gu N, Park MK, Kim TE, et al. Multiple-dose pharmacokinetics and pharmacodynamics of evogliptin (DA-1229), a novel dipeptidyl peptidase IV inhibitor, in healthy volunteers. Drug Des Devel Ther. 2014;8:1709-1721. doi: 10.2147/DDDT.S65678

25. Jung $\mathrm{CH}$, Park $\mathrm{CY}$, Ahn KJ, et al. A randomized, double-blind, placebo-controlled, phase II clinical trial to investigate the efficacy and safety of oral DA-1229 in patients with type 2 diabetes mellitus who have inadequate glycaemic control with diet and exercise. Diabetes Metab Res Rev. 2015;31(3):295-306. doi: $10.1002 / \mathrm{dmr} .2613$

26. Oh J, Kim AH, Lee S, et al. Effects of renal impairment on the pharmacokinetics and pharmacodynamics of a novel dipeptidyl peptidase-4 inhibitor, evogliptin (DA-1229). Diabetes Obes Metab. 2017;19(2):294-298. doi: 10.1111/dom.12813

27. Delarue J, Magnan C. Free fatty acids and insulin resistance. Curr Opin Clin Nutr Metab Care. 2007;10(2):142-148. doi: 10.1097/MCO.0b013e328042 ba90 


\section{ИНФОРМАЦИЯ ОБ АВТОРАХ [AUTHORS INFO]}

Бабенко Алина Юрьевна, д.М.н., доцент [Alina Y. Babenko, MD, PhD, associate professor]; адрес: Россия, 197341, Санкт-Петербург, ул. Аккуратова, д. 2 [address: 2, Akkuratova Street, Saint-Petersburg, 197341 Russian Federation]; ORCID: https://orcid.org/0000-0002-0559-697X; eLibrary SPIN: 9388-1077; e-mail: alina_babenko@mail.ru

Мосикян Анна Альбертовна [Anna A. Mosikian]; ORCID: https://orcid.org/0000-0002-2863-270X; eLibrary SPIN: 96056480; e-mail: mosikian.anna@gmail.com

Шляхто Евгений Владимирович, д.м.Н., профессор, академик PAH [Evgeny V. Shlyakhto, MD, PhD, Professor]; ORCID: https://orcid.org/0000-0003-2929-0980; eLibrary SPIN: SPIN-код: 6679-7621; e-mail: e.shlyakhto@almazovcentre.ru Макаренко Игорь Евгеньевич, к.м.н., медицинский научный советник [lgor E. Makarenko, PhD, medical advisor], ORCID: http://orcid.org/0000-0003-2308-0608; eLibrary SPIN: 8213-1789; e-mail: igor.makarenko@geropharm.com Леушева Виктория Витальевна [Victoriya V. Leusheva]; ORCID: https://orcid.org/0000-0001-5077-9858; eLibrary SPIN: 2000-5500; e-mail: Viktoriya.Leusheva@geropharm.com

\section{ЦИТИРОВАТЬ:}

Бабенко А.Ю., Мосикян А.А., Макаренко И.Е., Леушева В.В., Шляхто Е.В. Анализ эффективности и безопасности эвоглиптина по сравнению с ситаглиптином при добавлении к монотерапии метформином в русско-корейской популяции. Результаты исследования ЭВОКОМБИ // Сахарный диабет. — 2018. — Т. 21. — №4. — С. $241-254$. doi: 10.14341/DM9586

\section{TO CITE THIS ARTICLE:}

Babenko AY, Mosikian AA, Makarenko IE, Leusheva VV, Shlyakhto EV. Efficacy and safety of evogliptin versus sitagliptin as add on to metformin alone in a combined russian-korean population. Evo-combi trial. Diabetes Mellitus. 2018;21(4):241-254. doi: 10.14341/DM9586 